\title{
ASSESSING AND COMPARING THE PERFORMANCE OF ENDMEMBER EXTRACTION METHODS IN MULTIPLE CHANGE DETECTION USING HYPERSPECTRAL DATA
}

\author{
H. Jafarzadeh ${ }^{1}$, M. Hasanlou ${ }^{1, *}$ \\ ${ }^{1}$ School of Surveying and Geospatial Engineering, College of Engineering, University of Tehran, Tehran, Iran \\ (h.jafarzadeh73, hasanlou)@ut.ac.ir
}

KEY WORDS: Change Detection, Hyperspectral Images, Unmixing, Endmember, Remote Sensing

\begin{abstract}
:
Endmember extraction is a process to identify the hidden pure source signals from the mixture. Endmember finding has become increasingly important in hyperspectral data exploitation because endmembers can be used to specify unknown particular spectral classes. This paper evaluates the change detection problem in bi-temporal hyperspectral remote sensing images using the unmixing process. A complete spectral unmixing process contains estimating the number of endmembers, endmember extraction and abundance estimation. Endmember extraction is a vital step in spectral unmixing of hyperspectral images. Hyperspectral change detection by unmixing has the potential to provide subpixel information from hyperspectral images. In this study, four methods including Simplex Identification via variable Splitting and Augmented Lagrangian (SISAL), N-finder algorithm (N-FINDR), Vertex Component Analysis (VCA), and Fast algorithm for linearly Unmixing (FUN) are used to produce multiple change detection maps. This paper explores and compares the performance of these methods in multiple change detection. The empirical results reveal the superiority of the FUN method in providing multiple change map with an overall accuracy of $87 \%$ and a kappa coefficient of 0.70 .
\end{abstract}

\section{INTRODUCTION}

The availability of images acquired on the same geographical area by satellite sensors at different times makes it possible to identify and label possible changes that have occurred on the ground (Bovolo et al., 2007). Change detection (CD) is one of the most important uses of remote sensing, and it plays a key role in many applications (Jafarzadeh et al., 2019; Liu et al., 2015). The results of the $C D$ are generally available in two forms: (1) a binary change map (i.e., the separation of change or no-change pixels), or (2) a multiple change map (i.e., the detection of different kinds of change) (Hasanlou et al., 2018). Analyzing the impact of endmember extraction methods in detecting multiple changes is the topic of this research.

By the advent of new hyperspectral satellite sensors, hyperspectral images (HSIs) can be obtained by very high spectral resolution and each pixel is generally mixed by a number of materials present in the scene (D. C. Heinz, 2001), so CD task in HSIs is more challenging than multispectral images (Liu et al., 2017). Pixels containing more than one material are called mixed pixels (Arai et al., 2009), in contrast to pure pixels, which only contain one material.

To solve this problem, spectral unmixing techniques were developed, aiming to detect the materials (termed endmembers) in the mixed pixels and to estimate their corresponding fractions (termed abundances) (Liu et al., 2016). Thus, the hyperspectral unmixing is actually an inverse problem. Endmember extraction is a vital step in spectral unmixing of HSIs (Guerra et al., 2015). Endmembers refer to the pure materials' spectra in HSIs, and endmember extraction is a process of finding the spectra of all the endmembers (Guerra et al., 2015). During the last decade, several algorithms have been proposed for the purpose of endmember selection from hyperspectral scenes (Plaza et al., 2004).
CD by unmixing offers the important promise of providing the nature of the change, whether it is a change in the abundances of pure materials in the pixel or the addition/subtraction of an endmember to/from the scene (Ertürk et al., 2015). In the literature, there are several hyperspectral CD approaches using unmixing algorithms such as methods presented in (Ertürk et al., 2015) and (Liu et al., 2016).

\section{METHODOLOGY}

Hyperspectral unmixing involves estimating the number of endmembers (all or some of them), endmember extraction, and their abundances at each pixel (Bioucas-Dias et al., 2012). Researchers have devised and investigated many algorithms extracting endmembers (Guerra et al., 2015). In this paper, the procedure of spectral unmixing and obtaining multiple change maps are applied via following process.

\subsection{Estimating the number of endmembers and endmember extraction}

During the last decade, several algorithms have been proposed for the purpose of autonomous/supervised endmember selection from hyperspectral scenes. Similar to the approach proposed by (Ertürk et al., 2015), for estimating the number of endmembers we adopted using Hyperspectral Signal identification by the minimum error (HySime) algorithm (Bioucas-Dias et al., 2008). In continues for evaluating impact of endmember extraction algorithms in multiple $\mathrm{CD}$, we implemented following endmember extraction methods on data sets: Simplex Identification via variable Splitting and Augmented Lagrangian (SISAL) (Bioucas-Dias et al., 2012), N-finder algorithm (NFINDR) (Winter, 1999) and Vertex Component Analysis (VCA) (Nascimento et al., 2005). Also, we used the new and

\footnotetext{
* Corresponding author
} 
Fast algorithm for linearly Unmixing (FUN) presented in (Guerra et al., 2015) which simultaneously estimates the number of endmembers and extracts them. The VCA and the NFINDR algorithm are frequently used for the endmember extraction (Guerra et al., 2015) and the FUN algorithm is a novel method named for linearly unmixing HSIs.

2.1.1 SISAL: The SISAL algorithm is utilized widely in hyperspectral unmixing for endmember extraction. This method implements a robust version of the Minimum Volume (MV) concept (Bioucas-Dias et al., 2012). The robustness is introduced by allowing the positivity constraint to be violated. Due to the presence of noise, or any other perturbation source, the spectral vectors may lie outside the true data simplex. In order to estimate endmembers more accurately, SISAL allows violations to the positivity constraint. Violations are penalized using the hinge function (Bioucas-Dias et al., 2012).

2.1.2 N-FINDR: The N-FINDR method finds the set of pixels that define the simplex with the maximum volume, potentially inscribed within the data set. First, a dimensionality reduction of the original image is accomplished by using the maximum noise fraction (MNF) transform. Next, randomly selected pixels qualify as endmembers, and a trial volume is calculated for every pixel in each endmember position by replacing that endmember and recalculating the volume. If the replacement results in a volume increase, the pixel replaces the endmember. This procedure, which does not require any input parameters, is repeated until there are no replacements of endmembers left (Winter, 1999).

2.1.3 VCA: The VCA technique is one of the most advanced convex-geometry-based endmember detection methods with the pure-pixel assumption. Considering the variations due to the surface topography, VCA models the data using a positive cone, whose projection onto a properly chosen hyperplane is a simplex with vertices being the endmembers. After projecting the data onto the selected hyperplane, VCA projects all image pixels to a random direction and uses the pixel with the largest projection as the first endmember. The other endmembers are identified by iteratively projecting data onto a direction (Nascimento et al., 2005).

2.1.4 FUN: Based on the FUN algorithm, which has been recently proposed in (Guerra et al., 2015), we can estimate the number of endmembers and extract them simultaneously. The FUN algorithm has to be initialized with a pixel (as the first endmember). In order to find first pure pixel or to select the first endmember, the FUN algorithm selects the pixel of the HSI with the largest orthogonal projection to the average pixel (called centroid pixel) using the Modified Gram-Schmidt Orthogonalization method. After that, this algorithm sequentially performs the orthogonal projections of the HSI, so in every iteration, one endmember is found. In every iteration, before these extracted pixels to be considered as endmembers, the FUN algorithm calculates the percentage of information that would be lost if that endmember was not extracted, till the real endmembers to be extracted. In order to do that, a stop factor should be calculated and compared with an input value $\alpha$. So, if the stopping factor is lower than the input value, the pixel is not considered as endmember, and the algorithm stops. The stop factor is defined as: 'the norm of the $i$ th pixel divided by the norm of the Gram-Schmidt orthogonalization' multiplied by 100 (Guerra et al., 2015).

\subsection{Abundances estimation}

Linear spectral mixture analysis is a widely used technique in remote sensing to produce amounts of materials abundance present in an image pixel (Bioucas-Dias et al., 2012). Assume that there are $p$ endmembers in an image scene, and $\left[m_{1}, m_{2}, \ldots\right.$, $\left.m_{p}\right]$ are the signatures of these materials and $\left[\alpha_{1}, \alpha_{2}, \ldots, \alpha_{p}\right]$ are their associated abundance fractions. Obviously, an image scene may contain as many as materials, while a pixel may contain only one or a few materials. In order for a linear spectral mixture model to produce accurate amounts of signature abundance fractions, usually, two constraints are imposed on the abundance fractions of materials in a pixel: (1) abundance sum-to-one constraint and (2) abundance non-negativity constraint (D. C. Heinz, 2001). A fully constrained linear spectral mixture analysis method will simultaneously implement both of aforementioned constraints (D. C. Heinz, 2001).

A method that simultaneously implemented based on both aforementioned constraints has been proposed in (D. Heinz et al., 1999) which is called Fully Constrained Least Squares (FCLS) that can be used to solve for fully constrained linear mixing problems (D. C. Heinz, 2001).

In this step, the FCLS is employed on the endmembers extracted by SISAL, N-FINDR and VCA algorithms to get the abundance value for each endmember of images. On the other hand, abundances calculated for the endmembers extracted by the FUN algorithm based on technique explained in (Guerra et al., 2015).

\subsection{Multiple Change Detection}

The unmixing process using the aforementioned techniques is applied to each temporal data set separately and the two corresponding abundance vectors of each pixel are compared according the technique presented in (Liu et al., 2016), and multiple change maps obtained.

In order to compare the results obtained with the FUN algorithm with some of the most relevant algorithms in the state of the art for the same task (i.e., CD), the entire unmixing process has been also performed using the unmixing chain formed by the HySime as a method for estimation the number of endmembers, SISAL, N-FINDR and VCA as the methods for endmember extraction and finally using FCLS for abundances estimation, for the same images.

\section{STUDY AREA}

\subsection{Data sets}

In this work, we utilized two widely used real-world HSIs to evaluate the performance of the endmember extraction methods in $\mathrm{CD}$. These data sets are reference and benchmark data set that have been used previously in many hyperspectral CD approaches (Hasanlou et al., 2018; Liu et al., 2015)and can be found online in http://rslab.ut.ac.ir.

Figure 1 shows the first data set, covering farmland near the city of Yuncheng Jiangsu province in China. The data were acquired on May 3, 2006 and April 23, 2007, respectively. The changes in this data set are mostly derived from land cover changes, as well as many subtle changes. The size of this data set is $420 \times 140$. 


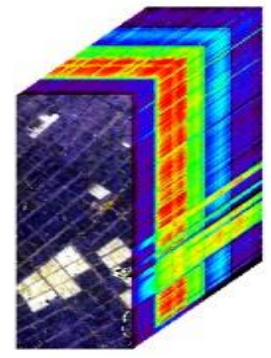

(a)

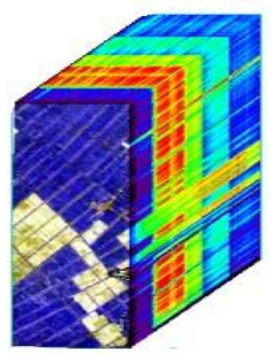

(b)

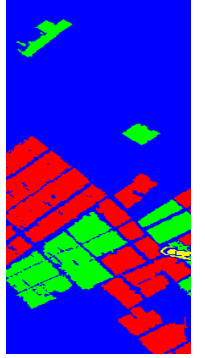

(c)
Figure 1. The (a) and (b) presented false-color composite of the original HSIs acquired in 2006 and 2007 of the China data set

respectively, and (c) multiple change map ground truth.

The second case study area covers a range of $307 \times 241$ of an irrigated agricultural field in the city of Hermiston in Umatilla County, Oregon, USA. The data were acquired on May 1, 2004, and May 8, 2007 (Figure 2). The main changes between the acquisition dates relate to land cover changes in agricultural fields.

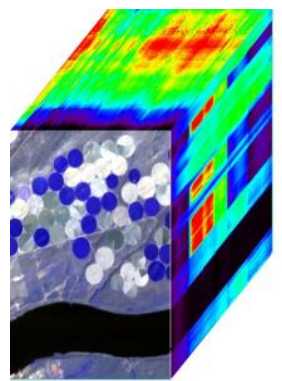

(a)

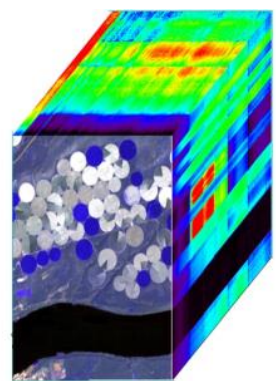

(b)

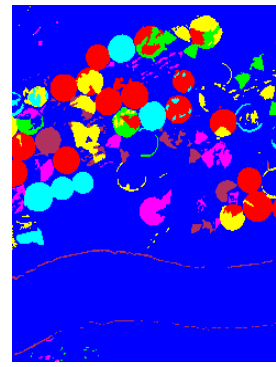

(c)
Figure 2. The (a) and (b) presented false-color composite of the original HSIs acquired in 2004 and 2007 of the USA data set respectively, and (c) multiple change map ground truth.

\subsection{Pre-processing}

Due to environmental and equipment conditions, data preprocessing plays a crucial role in remote sensing before the beginning of the main process. The pre-processing is made in two steps: (1) geometric pre-processing and (2) spectral preprocessing (including remove no-data bands, de-striping, noise reduction, smile-frown detection, radiometric calibration, and atmospheric correction) (Ertürk et al., 2015; Hasanlou et al., 2018; Jafarzadeh et al., 2019).

\section{RESULTS AND DISCUSSION}

The multiple change maps obtained based on unmixing procedure for the China data set are depicted in Figure 3. In addition, we assessed and compared (visually and numerical) the performance of the aforementioned techniques in CD based on obtained results. Visually comparing of the multiple change maps presented in Figure 3 with the multiple change map ground truth shows that the change map obtained based on the FUN algorithm is closer to reality. To prove this fact numerical analysis of multiple change maps presented in Table 1.

For numerical analyzing, we used three indicators including Overall Accuracy (OA), Kappa Coefficient $(\kappa)$ and False Positive Rate (FPR) to assess the multiple change maps.

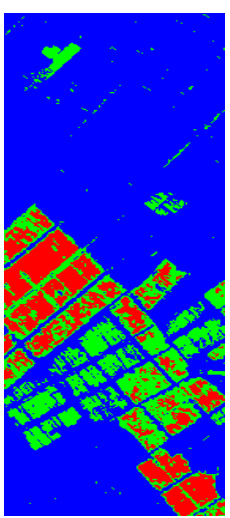

(a)

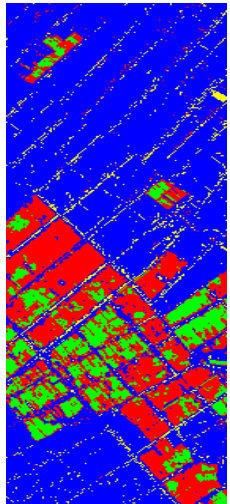

(b)

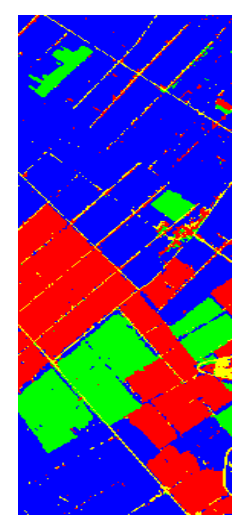

(c)

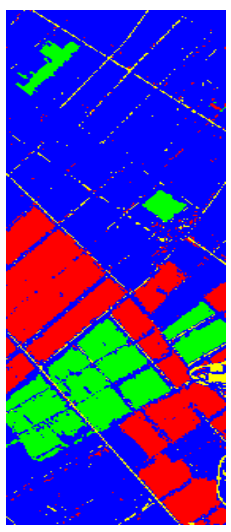

(d)

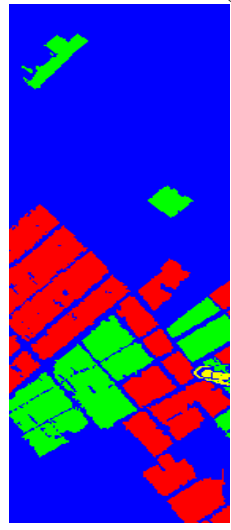

(e)
Figure 3. The result of multiple change maps detected by different endmember extraction algorithms for China data set. (a) SISAL, (b) N-FINDR, (c) VCA, (d) FUN, and (e) multiple change map ground truth.

Table 1. Numerical evaluation of different hyperspectral CD methods in China data set.

\begin{tabular}{lccc}
\hline \multirow{2}{*}{ Method } & \multicolumn{3}{c}{ Indicator } \\
\cline { 2 - 4 } & $\mathrm{OA}(\%)$ & $\kappa$ & FPR \\
\hline SISAL & 83 & 0.63 & 0.09 \\
N-FINDR & 83 & 0.56 & 0.16 \\
VCA & 87 & 0.66 & 0.04 \\
FUN & $\mathbf{9 0}$ & $\mathbf{0 . 7 3}$ & $\mathbf{0 . 0 4}$ \\
\hline
\end{tabular}

According to Figure 3 and Table 1, the change map obtained based on the FUN algorithm represents a more advantageous in endmember extraction than the algorithms of the state of the art since this algorithm reaches a high accuracy in multiple CD. The SISAL, N-FINDR, and VCA based unmixing CD have different results while all of these methods used the same endmember estimation and employed one algorithm to get the abundance value for each endmember. This issue shows the importance of endmember extraction methods in hyperspectral CD.

Moreover, hyperspectral CD with the aim of unmixing for USA data set implemented. The results of multiple change maps presented in Figure 4. Visually comparing of the multiple change maps with the multiple change map ground truth shows that the change map obtained using FUN algorithm is closer to ground truth. 


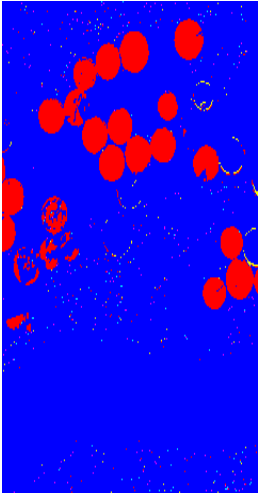

(a)

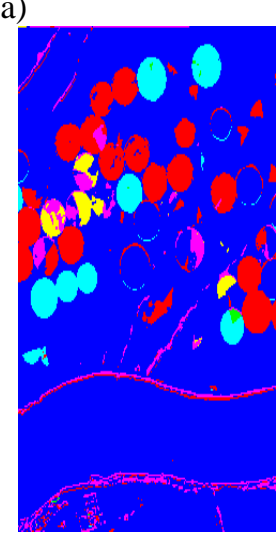

(d)

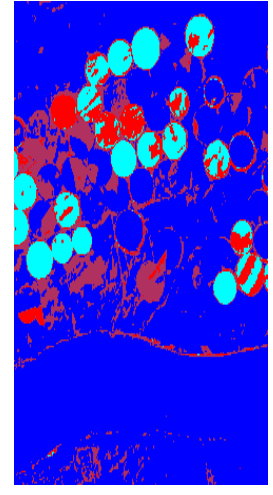

(b)

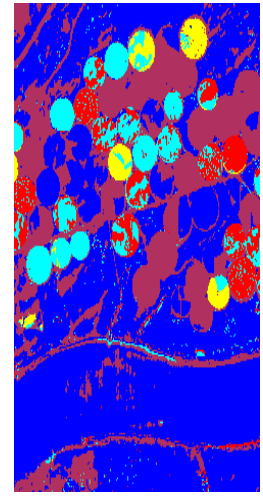

(c)

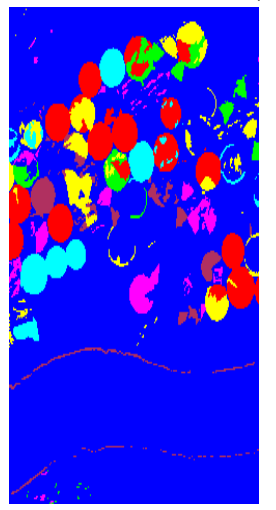

(e)

Figure 4. The result of multiple change maps detected by different endmember extraction algorithms for USA data set. (a) SISAL, (b) N-FINDR, (c) VCA, (d) FUN, and (e) multiple change map ground truth.

The value of three indicators to assess the multiple change maps is presented in Table 2.

Table 2. Numerical evaluation of different hyperspectral CD methods in the USA data set.

\begin{tabular}{lccc}
\hline \multirow{2}{*}{ Method } & \multicolumn{3}{c}{ Indicator } \\
\cline { 2 - 4 } & $\mathrm{OA}(\%)$ & $\kappa$ & FPR \\
\hline SISAL & 87 & 0.59 & 0.09 \\
N-FINDR & 77 & 0.40 & 0.08 \\
VCA & 58 & 0.22 & 0.12 \\
FUN & $\mathbf{8 7}$ & $\mathbf{0 . 7 0}$ & $\mathbf{0 . 0 4}$ \\
\hline
\end{tabular}

The experimental results demonstrate that the accuracy of the change map obtained by the FUN algorithm is better than the accuracy of the change maps obtained by the other methods. In other words, the accuracy obtained with the FUN algorithm when extracting endmembers and calculating abundances is better than the accuracy of the SISAL, N-FINDR and VCA algorithms when extracting endmembers and calculating abundances of them by FCLS method.

\section{CONCLUSION}

This work shows the importance of endmember extraction methods in hyperspectral CD. A complete spectral unmixing process contains endmember extraction and abundance estimation. There are various endmember extraction algorithms

which are applicable to the hyperspectral CD. A set of experiments with real data sets led to the conclusion that the FUN algorithm is an effective and useful tool, yielding comparable or better results than some of the most relevant endmember extraction algorithms. The experiments demonstrated that the accuracy obtained with FUN algorithm when extracting the endmembers is better than the accuracy of SISAL, N-FINDR, and VCA algorithms. Hence, the FUN algorithm represents a more advantages solution than the algorithms of the state of the art and can be widely used in CD approaches.

\section{REFERENCES}

Arai, K. and Chen, H., 2009. Unmixing method for hyperspectral data based on sub-space method with learning process. Advances in Space Research, 44(4), 517-523.

Bioucas-Dias, J. M. and Nascimento, J. M., 2008. Hyperspectral subspace identification. IEEE Transactions on Geoscience Remote Sensing, 46(8), 2435-2445.

Bioucas-Dias, J. M., Plaza, A., Dobigeon, N., Parente, M., Du, Q., Gader, P., and Chanussot, J., 2012. Hyperspectral unmixing overview: Geometrical, statistical, and sparse regression-based approaches. IEEE journal of selected topics in applied earth observations remote sensing, 5(2), 354-379.

Bovolo, F. and Bruzzone, L., 2007. A theoretical framework for unsupervised change detection based on change vector analysis in the polar domain. IEEE Transactions on Geoscience Remote Sensing, 45(1), 218-236.

Ertürk, A. and Plaza, A., 2015. Informative change detection by unmixing for hyperspectral images. IEEE Geoscience Remote Sensing Letters, 12(6), 1252-1256.

Guerra, R., Santos, L., López, S., and Sarmiento, R., 2015. A new fast algorithm for linearly unmixing hyperspectral images. IEEE Transactions on Geoscience Remote sensing of environment, 53(12), 6752-6765.

Hasanlou, M. and Seydi, S. T., 2018. Hyperspectral change detection: An experimental comparative study. International Journal of Remote Sensing, 39(20), 7029-7083.

Heinz, D., Chang, C.-I., and Althouse, M. L., 1999. Fully constrained least-squares based linear unmixing [hyperspectral image classification]. Paper presented at the IEEE 1999 International Geoscience and Remote Sensing Symposium. IGARSS'99 (Cat. No. 99CH36293).

Heinz, D. C., 2001. Fully constrained least squares linear spectral mixture analysis method for material quantification in hyperspectral imagery. IEEE transactions on geoscience remote sensing, 39(3), 529-545.

Jafarzadeh, H. and Hasanlou, M., 2019. Probability Estimation of Change Maps Using Spectral Similarity. Paper presented at the Multidisciplinary Digital Publishing Institute Proceedings.

Liu, S., Bruzzone, L., Bovolo, F., and Du, P., 2015. Hierarchical unsupervised change detection in multitemporal hyperspectral images. IEEE Transactions on Geoscience Remote sensing of environment, 53(1), 244-260. 
Liu, S., Bruzzone, L., Bovolo, F., and Du, P., 2016. Unsupervised multitemporal spectral unmixing for detecting multiple changes in hyperspectral images. IEEE Transactions on Geoscience Remote Sensing, 54(5), 2733-2748.

Liu, S., Tong, X., Bruzzone, L., and Du, P., 2017. A novel semisupervised framework for multiple change detection in hyperspectral images. Paper presented at the IEEE International Geoscience and Remote Sensing Symposium (IGARSS).

Nascimento, J. M. and Dias, J. M., 2005. Vertex component analysis: A fast algorithm to unmix hyperspectral data. IEEE transactions on Geoscience Remote Sensing, 43(4), 898-910.

Plaza, A., Martínez, P., Pérez, R., and Plaza, J., 2004. A quantitative and comparative analysis of endmember extraction algorithms from hyperspectral data. IEEE transactions on geoscience remote sensing, 42(3), 650-663.

Winter, M. E., 1999. N-FINDR: An algorithm for fast autonomous spectral end-member determination in hyperspectral data. Paper presented at the Imaging Spectrometry V. 